\title{
Anti-atherosclerotic effect of traditional fermented cheese whey in atherosclerotic rabbits and identification of probiotics
}

\author{
Xin-Hua Nabi", Chun-Yan Ma, Tabusi Manaer, Mulalibieke Heizati, Baheti Wulazibieke and Latipa Aierken
}

\begin{abstract}
Background: Traditional fermented cheese whey (TFCW), containing probiotics, has been used both as a dairy food with ethnic flavor and a medicine for cardiovascular disease, especially regulating blood lipid among Kazakh. We therefore investigated anti-atherosclerotic effects of TFCW in atherosclerotic rabbits and identified lactic acid bacteria $(\mathrm{LAB})$ and yeasts in TFCW.

Methods: Atherosclerotic rabbits were induced by administration of atherosclerotic diet for 12 weeks and divided randomly into three groups and treated for 4 weeks with Simvastatin $(20 \mathrm{mg} / \mathrm{kg}$ ) or TFCW $(25 \mathrm{mg} / \mathrm{kg})$ and $(50 \mathrm{mg} / \mathrm{kg})$. In addition, a normal control group and an atherosclerotic group were used for comparison. All drugs were intragastrical administered once daily $10 \mathrm{~mL} / \mathrm{kg}$ for 4 weeks. Body weight (BW), lipid profiles, C-reactive protein (CRP), vascular cell adhesion molecule-1 (VCAM-1), and intercellular adhesion molecule-1 (ICAM-1) were tested and theromatous plaques and the number of foam cells and infiltrating fibroblast cells in the thoracic aorta endothelium was evaluated by hematoxylin and eosin stainin. $\angle A B$ and yeasts were isolated and purified by conventional techniques and identified using morphological and biochemical properties as well as gene sequences analysis.
\end{abstract}

Results: After 4 weeks of treatment, high and low dose TFCW decreased serum TC, TG, LDLC, CRP, VCAM-1 and ICAM-1 $(P<0.05)$ compared to atherosclerotic group, and increased HDL-C $(P<0.05)$ compared to normal controls. Histological analysis showed TFCW reduced VCAM-1 expression and formation of atheromatous plaques on the aortic endothelium of atherosclerotic rabbits.

Conclusion: Seven classes of LBA from two different genera including Lactobacillus brevis, Lactobacillus kefianofaciens, Lactobacillus helveticus, Lactobacillus Casei, Lactobacillus plantarum, Lactobacillus kefiri and Lactococcus lactic as well as 2 classes of yeasts from two different genera including Saccharomyces unisporus and Issatchenkia orientalis were isolated and identified from TFCW. In summary, TFCW, containing 7 classes of LBA and 2 classes of yeasts, has significant anti-atherosclerotic potential in atherosclerotic rabbits and may modulate lipid metabolism and protect aorta in the atherosclerotic condition, which might be related to various probiotics acting through reducing the CRP, VCAM-1 and ICAM-1 levels and protecting the aortic endothelium.

Keywords: Traditional fermented cheese whey, Atherosclerosis, Vascular cellular adhesion molecule-1, Intercellular cellular adhesion molecule-1, C-reactive protein, Lipid profile, Lactic acid bacteria, Yeast

Abbreviations: BW, Body weight; CRP, C-reactive protein; CVD, Cardiovascular disease; HDL-C, High density lipoprotein cholesterol; HPF, High power field; HTST, High temperature short time; ICAM-1, Intercellular adhesion molecule-1; LAB, Lactic acid bacteria; LDL-C, Low-density lipoprotein cholesterol; MRS, Man Rogosa Sharpe; PPAR-Y, Peroxisome proliferator-activated receptor-y; SPF, Specific pathogen free; TC, Total cholesterol; TFCW, Traditional fermented cheese whey; TG, Triglycerides; VCAM-1, Vascular cell adhesion molecule-1

\footnotetext{
* Correspondence: xinhua99@hotmail.com

Department of Pharmacology, Xinjiang Medical University, Urumqi 830011,

China
} 


\section{Background}

The public health burden of cardiovascular disease (CVD) is substantial, as CVD remains the leading cause of mortality and morbidity worldwide and atherosclerosis is the major cause of CVD $[1,2]$.

Traditional fermented cheese whey (TFCW), by-product of cheese-making, has been widely used as a traditional dairy medication for regulating blood lipid among Kazakh people [3]. Indeed, active peptides in TFCW up-regulate expression of peroxisome proliferator-activated receptor- $\gamma$ (PPAR- $\gamma$ ) mRNA [3], which reduces atherosclerosis [4]. Furthermore, whey protein and peptides have a protective effect against CVD risk factors [5].

Probiotics mainly include Lactobacillus and Bifidobacterium and a few yeast species including Saccharomyces boulardi [6]. Lactic acid bacteria (LAB) are the main probiotics that prevent formation of aortic fatty lesions by inhibiting low-density lipoprotein (LDL) oxidation [7] and atherosclerosis via the inhibition of intestinal cholesterol absorption [8] in animal models. S. boulardii, one of the probiotic yeasts, provides anti-inflammatory and host immunity stimulatory effects [9] and lowers remnant lipoprotein, a highly atherogenic lipoprotein particle, in human adults with hypercholesterolemia [10].

However, anti-atherosclerotic effects of TFCW have not been experimentally demonstrated and no LAB or yeast has been found in TFCW. The aims of this study were to investigate anti-atherosclerotic effects of TFCW in a rabbit model of atherosclerosis and to identify LBA and yeast in TFCW.

\section{Methods}

Traditional fermented cheese whey (TFCW) manufacturing Traditional fermented cow's milk is the source of the cheese whey. Experimental TFCW samples were manufactured by standard procedures in $10 \mathrm{~L}$ vats in Altay Kanas Dairy Co. Ltd., (Altay, Xinjiang, China). Fresh cow's milk samples were obtained from Jimunai Saur farm (Altay, Xinjiang, China) and skimmed in centrifuging at $3000 \times \mathrm{g}$ for $30 \mathrm{~min}$, homogenized under the pressure of $1.5 \sim 1.7$ Mpa and pasteurized by high temperature short time (HTST) then cooled to about $30{ }^{\circ} \mathrm{C}$ and fermented by inoculation with traditional home made Kazak yogurt purchased from Jimunai Saur farm (Altay, Xinjiang, China) at $37{ }^{\circ} \mathrm{C}$ for $12 \mathrm{~h}$. After ferment, the whey was filtered in sterile gauze and dialyzed in cellulose membrane (12 kDa, Sigma) under constant magnetic stirring at $8{ }^{\circ} \mathrm{C}$, also performed lactose removal by periodic water exchange. The experimental TFCW was stored at $-20{ }^{\circ} \mathrm{C}$ until further use.

\section{Chemicals and reagents}

Sodium pentobarbital was purchased from Merck \& Co., (Germany). Simvastatin was purchased from Merck Sharp
\& Dohme (Australia) Pty Ltd., (Hangzhou, China). VCAM1, ICAM-1 and CRP ELISA kits were purchased from Shanghai Senxiong Technology Co. Ltd., (Shanghai, China). Man Rogosa Sharpe (MRS) was purchased from Merck Sharp \& Dohme (Australia) Pty Ltd., (Hangzhou, China). All media for cultivation of zymocytes were purchased from Hangzhou Microbial Reagent Co. Ltd., (Hangzhou, China).

\section{Animals and treatment}

Sixty male white New Zealand rabbits, weighing 1.95$2.05 \mathrm{~kg}$, specific pathogen free (SPF), were provided by Experimental Animal Center of Xinjiang Medical University, China and placed in separate cages and maintained on a 12-h day/night cycle at an ambient temperature, with ad libitum access to food and water. After a week of adaptive feeding, all the rabbits were randomly divided into 5 groups with 12 in normal group and 12 in atherogenic group, the normal control groups were given regular die and the atherogenic models were developed using an atherogenic diet for 12 weeks. The atherogenic diet consisted of $3 \%$ cholesterol, $0.5 \%$ sodium taurocholate, $0.2 \%$ propylthiouracil, $5 \%$ sugar, $10 \%$ lard, and $81.3 \%$ standard laboratory rabbit chow, which were provided by Experimental Animal Center of Xinjiang Medical University, China. After developing atherogenic models, Group 1 (normal control) was treated with saline in a matched volume; Group 2 (atherogenic group) had atherogenic rabbits treated with saline in a matched volume; Group 3 (positive control) had atherogenic rabbits administered with simvastatin $20 \mathrm{mg} / \mathrm{kg}$; Group 4 and Group 5 were treated with TFCW $25 \mathrm{mg} / \mathrm{kg}$ and $50 \mathrm{mg} / \mathrm{kg}$, respectively (low and high doses). Simvastatin and TFCW were intragastrical administered once daily $10 \mathrm{~mL} / \mathrm{kg}$ for 4 weeks. All animals received care in compliance with the Chinese Convention on Animal Care, and the study was approved by the Institutional Ethics Committee of Xinjiang Medical University.

\section{Collection of blood and biochemical measurement}

At the end of experiments, all rabbits were fasted for $12 \mathrm{~h}$, weighed, anesthetized with sodium pentobarbital (Merck \& Co.,) and continually monitored until total loss of consciousness as indicated by a total lack of response after a foot pinch. Blood samples were collected from abdominal aorta, allowed to clot on ice and subsequently subjected to centrifugation $\left(3500 \mathrm{rpm}\right.$ at $4{ }^{\circ} \mathrm{C}$ for $10 \mathrm{~min}$ ), where after serum aliquots were stored at $-80{ }^{\circ} \mathrm{C}$ for further analysis. Serum TC, TG, LDL-C and HDL-C were examined via an automatic biochemical analyzer (BS-120, Shenzhen Mindray High-Tech Co., Ltd. China). CRP was determined by rate nephelometry (Beckman Coulter, USA). Serum ICAM-1 and VCAM-1 were determined using commercially-available ELISA kits according to manufacturer instruction. 


\section{Histopathological study of aorta}

Aorta was harvested from rabbits, placed immediately in formaldehyde $10 \%$, embedded in paraffin $24 \mathrm{~h}$ later, cut at $5 \mu \mathrm{m}$, stained with hematoxylin and eosin (H\&E), and then scanned to assess pathological changes. For immunohistochemical staining, sections were incubated with anti-VCAM-1 (R\&D Systems, MN, USA) and antiF4/80 (Abcam, MA, USA) at $37{ }^{\circ} \mathrm{C}$ for $1 \mathrm{~h}$, color developed with 3,3'-diaminobenzidine tetrahydrochloride and counterstained with hematoxylin. Samples in the absence of the primary antibodies were used as negative controls. Slides were observed under a light microscope, and images were subjected to statistical evaluation of positively stained cells in 10 random fields of view at a magnification of $\times 400$. The average numbers of positively stained cells were counted per high power field (HPF).

Isolation, purification and characterization of LAB

Agar plates with Man Rogosa Sharpe (MRS) broth suitable for lactobacillus growth were used for initial isolation of LAB single colonies. Single bacterial colonies were initially separated based on their morphological differences on agar plates. Cell morphology was observed under light microscopy after Gram staining. Catalase activity, carbohydrate fermentation, acidogenicity, aciduricity (final $\mathrm{pH}$ ), and gas $\left(\mathrm{CO}_{2}\right)$ production were analyzed. All isolates were presumptively identified as LAB strains based on their ability to grow on MRS agar plates, Gram-positive staining, and a catalase activity-negative phenotype [11]. 16S rDNA and 16S rRNA of 7 isolates were initially analyzed by BLAST program on NCBI website to search for the best matches among existing data in GenBank. 16S rDNA and $16 \mathrm{~S}$ rRNA gene sequence analyses were carried out at Institute of Microbiology, Chinese Academy of Sciences, Beijing, China.

\section{Isolation, purification and identification of yeasts}

Each $100 \mu \mathrm{l}$ sample was enriched in a tube containing Sabourauds agar medium, incubated at $25{ }^{\circ} \mathrm{C}$ for $48-72 \mathrm{~h}$ and spread on Sabourauds agar. Representative yeast colonies were selected based on colonial characteristics, purified using a single colony isolation method, and maintained on a Sabourauds agar slant at $4{ }^{\circ} \mathrm{C}$ or in freezing tubes containing Sabourauds agar broth supplemented with $10 \%$ glycerol at $-80{ }^{\circ} \mathrm{C}$. Physiological and biochemical characteristic identifications were made according to results of carbohydrate fermentation, carbon source assimilation, nitrogen assimilation and temperature tests. All these tests and analyses of 26S rDNA D1/D2 gene sequences were performed using identical methods as those used for bacteria.

\section{Statistical analysis}

All values were reported as mean \pm S.E.M. Data were analyzed by one-way ANOVA using SPSS 18 (SPSS Inc., Chicago, Illinois, USA). Significance was defined as " $P<$ 0.05 compared to atherogenic group.

\section{Results}

\section{Effects of TFCW on Lipid Profiles}

Table 1 showed serum lipid profiles among different experimental groups. Serum TC, TG, HDL-C and LDL-C of rabbits were significantly higher in atherogenic group than in normal control group $(p<0.05)$, whereas Simvastatin group and low and high dose TFCW group showed significantly lower serum TC, TG and LDL-C than did atherogenic group $(p<0.05)$. TFCW significantly increased HDL-C levels compared to normal control group $(p<0.05)$, but no significant difference was observed in treated groups with TFCW, compared with atherogenic group. Data indicated that TFCW affects lipid metabolic parameters and TFCW treatment could effectively improve lipid metabolism in atherogenic rabbits.

\section{Effects of TFCW on CRP levels and body weight}

Serum CRP was measured to determine inflammatory status of experimental groups as shown in Table 2. Generally, atherogenic group had significantly higher CRP, compared with the normal control group $(15.03 \pm 9.12$ vs $0.98 \pm 0.03 \mathrm{mg} / \mathrm{L}, P<0.05)$. Outstandingly, the simvastatin and low and high dose TFCW groups had significantly lower CRP $(P<0.05)$ at values $3.43 \pm 0.80 \mathrm{mg} / \mathrm{L}$, $3.33 \pm 0.50 \mathrm{mg} / \mathrm{L}$ and $1.34 \pm 0.90 \mathrm{mg} / \mathrm{L}$, respectively, compared with the atherogenic group. CRP in low and

Table 1 Effect of TFCW on TC, TG, LDL-C and HDL-C in atherosclerotic rabbits (mmol/L, $n=12$ )

\begin{tabular}{lllll}
\hline Group & TC & TG & HDL-C & LDL-C \\
\hline Normal control & $4.75 \pm 2.00$ & $0.53 \pm 0.16$ & $1.03 \pm 0.22$ & $0.18 \pm 0.03$ \\
Atherogenic group & $22.79 \pm 2.13^{*}$ & $3.20 \pm 1.32^{*}$ & $2.20 \pm 0.95^{*}$ & $15.85 \pm 3.2^{*}$ \\
Simvastatin $(20 \mathrm{mg} / \mathrm{kg})$ & $17.55 \pm 1.6^{* *}$ & $0.86 \pm 0.22^{* *}$ & $2.55 \pm 0.23^{*}$ & $7.23 \pm 2.87^{* *}$ \\
TFCW $(25 \mathrm{mg} / \mathrm{kg})$ & $16.74 \pm 3.4^{* *}$ & $1.49 \pm 0.73^{* *}$ & $2.16 \pm 0.69^{*}$ & $5.52 \pm 1.76^{* *}$ \\
TFCW $(50 \mathrm{mg} / \mathrm{kg})$ & $15.20 \pm 5.25^{* *}$ & $0.50 \pm 0.20^{* *}$ & $2.33 \pm 0.18^{*}$ & $6.7 \pm 2.28^{* *}$ \\
\hline
\end{tabular}

${ }^{*} P<0.05$ vs. Normal control

${ }^{* *} P<0.05$ vs. Atherogenic group 
Table 2 Effect of TFCW on ICAM-1, VCAM-1, CRP and bodyweight in atherosclerotic rabbits $(n=12)$

\begin{tabular}{lllll}
\hline Group & CRP $(\mathrm{mg} / \mathrm{L})$ & Body weight $(\mathrm{kg})$ & ICAM-1 $(\mu \mathrm{g} / \mathrm{L})$ & $\mathrm{VCAM}-1(\mu \mathrm{g} / \mathrm{L})$ \\
\hline Normal control & $0.98 \pm 0.03$ & $3.21 \pm 0.37$ & $32.89 \pm 16.00$ & $16.90 \pm 6.03$ \\
Atherogenic group & $15.03 \pm 9.12^{*}$ & $3.03 \pm 0.63$ & $118.50 \pm 30.12^{*}$ & $51.23 \pm 9.00^{*}$ \\
Simvastatin $(20 \mathrm{mg} / \mathrm{kg})$ & $3.43 \pm 0.80^{* *}$ & $2.90 \pm 0.15$ & $63.65 \pm 28.98^{* *}$ & $41.14 \pm 7.81^{* *}$ \\
TFCW $(25 \mathrm{mg} / \mathrm{kg})$ & $3.33 \pm 0.50^{* *}$ & $2.35 \pm 0.30$ & $75.60 \pm 28.40^{* *}$ & $41.12 \pm 8.90^{* *}$ \\
TFCW $(50 \mathrm{mg} / \mathrm{kg})$ & $1.34 \pm 0.90^{* *}$ & $3.03 \pm 0.30$ & $42.25 \pm 17.80^{* *}$ & $41.75 \pm 12.32^{* *}$ \\
\hline
\end{tabular}

${ }^{*} P<0.05$ vs. Normal control

${ }^{*} P<0.05$ vs. Atherogenic group

high dose TFCW was significantly decreased, compared to atherogenic group $(P<0.05)$. Data indicate that TFCW affects CRP and TFCW treatment could effectively improve inflammatory status in atherogenic rabbits. No significant differences were recorded in treated groups with TFCW on body weight compared with atherogenic group.

\section{Effects of TFCW on ICAM-1 and VCAM-1}

Adhesion molecules ICAM-1 and VCAM-1 play important roles in perpetuation of inflammation. Table 2 showed serum ICAM-1 and VCAM- 1 among different experimental groups. ICAM-1 and VCAM-1 of rabbits were significantly higher in atherogenic group than in normal control group $(p<0.05)$, while Simvastatin and low and high dose TFCW group showed significantly decreased ICAM-1 and VCAM-1, compared to atherogenic group $(p<0.05)$. Data indicated that TFCW might affect ICAM-1 and VCAM-1 and TFCW treatment might effectively inhibit adhesion of circulating inflammatory cells to endothelial cell walls in atherogenic rabbits.

Effects of TFCW on histopathological changes in the aorta Histopathological examination of aorta sections from experimental groups were shown in Fig. 1. Histological examination of hematoxylin and eosin-stained section of aorta of normal control showed normal histological examination of aorta, with no aortic lesion or presence of foam cells, while the aorta examination of atherogenic group showed thickening of intimal layer and accumulation of lipids, leading to formation of foam cells, signaling the early phase in development of atherosclerosis. Aorta of groups treated with simvastatin and low and high dose TFCW group showed reduced accumulation of atherosclerotic lesion due to decreased number of foam cells and cholesterol deposits in the aorta. Morphometric analysis of aorta section had showed that thickening of intimal layer and foam cells was increased in atherogenic group, compared to normal control group. Furthermore, groups treated with simvastatin and low and high dose TFCW showed decreased intimal layer and foam cells, compared with atherogenic group.
Effects of TFCW on immunohistochemistry assessment in the aorta

Immunohistochemical evaluation of VCAM-1 expression in aorta section and histomorphometric analysis from experimental groups were shown in Fig. 2. Atherogenic group showed strong VCAM-1 staining, compared to normal control group. Simvastatin and low and high dose TFCW groups showed less VCAM-1 expression than did atherogenic group, which indicated that TFCW may have a preventive effect in atherogenic rabbits.

\section{Identification of LAB}

Seven gram-positive, catalase-negative bacilli were isolated from TFCW. Most of the bacilli had yellow or white colonies with uneven edges and a rough and dull surface. Some of the colonies in the center were translucent, with neat edges. The type colony size was approximately 0.5 $3.0 \mathrm{~mm}$. Morphology of the separated Lactobacillus species was rod-shaped, slender short or long, and the majority of Lactobacillus exhibited chain-like arrangement. We successfully isolated and purified seven isolates of LBA and identified them as L. brevis, L. kefianofaciens, L. helveticus, L. casei, L. plantarum, L. kefiri and Lactococcus lactic based on morphological characteristics, physiological tests, biochemical tests, and 16S rDNA and 16S rRNA sequence homology with NCBI Reference Sequences NC 008497.1, NC 015602.1, NC 006814.3, NC 008526.1, NC 004567.2, NC 015428.1 and NC 017486. All the test results and sequence homology were shown in Table 3, while all of the sequences were as follows:

\section{L. brevis:}

GCTGACTCCCGAAGGTTATCTCACCGGCTTT GGGTGTTACAAACTCTCATGGTGTGACGGGC GGTGTGTACAAGGCCCGGGAACGTATTCACC GCGGCATGCTGATCCGCGATTACTAGCGATT CCAACTTCATGTAGGCGAGTTGCAGCCTACA ATCCGAACTGAGAACGGCTTTAAGAGATTAG CTTAGCCTCACGACTTCGCGACTCGTTGTAC CGTCCATTGTAGCACGTGTGTAGCCCAGGTC ATAAGGGGCATGATGATTTGACGTCATCCCC ACCTTCCTCCGGTTTGTCACCGGCAGTCTCA 

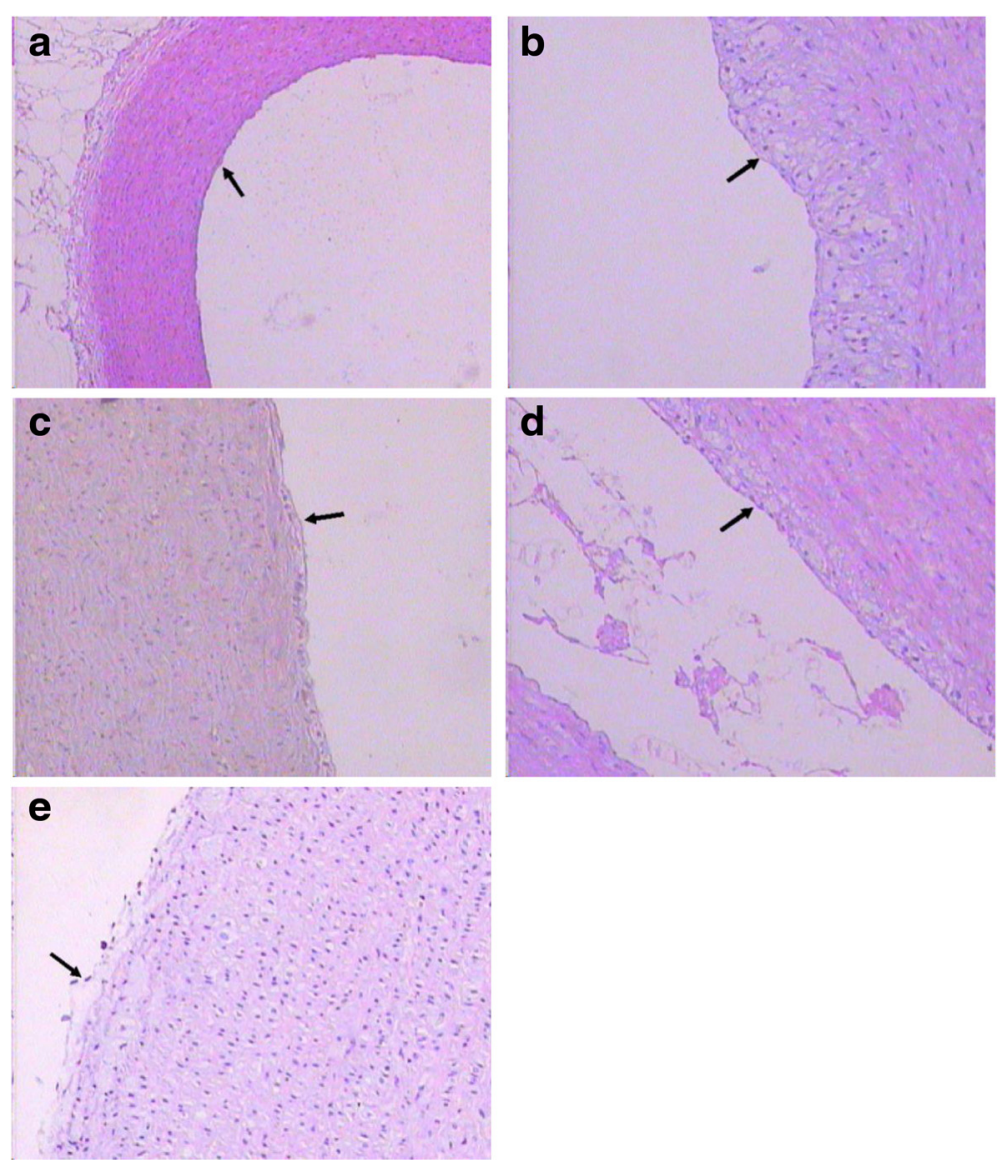

Fig. 1 The effect of TFCW on histological sections of aorta in atherosclerotic rabbits (H\&E). a Normal control; (b) atherosclerotic group; (c) positive control (simvastatin $20 \mathrm{mg} / \mathrm{kg}$ ); (d) low dose TFCW (25 mg/kg); (e) high dose TFCW (50 mg/kg). (Magnification 400x)

CCAGAGTGCCCAACTGAATGCTGGCAACTG ATAATAAGGGTTGCGCTCGTTGCGGGACTTA ACCCAACATCTCACGACACGAGCTGACGAC AACCATGCACCACCTGTCATTCTGTCCCCGA AGGGAACGTCTTATCTCTAAGATTGGCAGAA GATGTCAAGACCTGGTAAGGTTCTTCGCGTA GCTTCGAATTAAACCACATGCTCCACCGCTT GTGCGGGCCCCCGTCAATTCCTTTGAGTTTC AACCTTGCGGTCGTACTCCCCAGGCGGAGTG CTTAATGCGTTAGCTGCAGCACTGAAGGGCG GAAACCCTCCAACACTTAGCACTCATCGTTT ACGGCATGGACTACCAGGGTATCTAATCCTG TTCGCTACCCATGCTTTCGAGCCTCAGCGTC AGTTACAGACTAGACAGCCGCCTTCGCCACT GGTGTTCTTCCATATATCTACGCATTCCACCG CTACACATGGAGTTCCACTGTCCTCTTCTGC ACTCAAGTCTCCCAGTTTCCGATGCACTTCT CCGGTTAAGCCGAAGGCTTTCACATCAGACT TAAAAAACCGCCTGCGCTCGCTTTACGCCCA ATAAATCCGGACAACGCTTGCCACCTACGTAT TACCGCGGCTGCTGGCACGTAGTTAGCCGTG
GCTTTCTGGTTAAATACCGTCAACCCTTGAA CAGTTACTCTCAAAGGTGTTCTTCTTTAACAA CAGAGTTTTACGAGCCGAAACCCTTCTTCAC TCACGCGGCATTGCTCCATCAGACTTTCGTC CATTGTGGAAGATTCCCTACTGCTGCCTCCC GTAGGAGTTTGGGCCGTGTCTCAGTCCCAAT GTGGCCGATTACCCTCTCAGGTCGGCTACGT ATCATCGTCTTGGTGGGCCTTTACCTCACCA ACTAACTAATACGCCGCGGGATCATCCAGAA GTGATAGCCGAANCCACCTTTCAAACAAAAT CCATGCGGATTTTGTTGTTATACGGTATTAGC ACCTGTTTCCAAGTGTTATCCCCTGCTTCTG GGCAGATTTCCCACGTGTTACTCACCAGTTC GCCACTCGCTTCATTGTTGAAATCAANGCAA GCACGTCATTCAACGGAAGCTCGTTCGACT

2. L. kefianofaciens:

CTGCTTAGACGGCTCCTTCCTTGCGGTTAGG CCACCGGCTTTGGGCATTGCAGACTCCCATG GTGTGACGGGCGGTGTGTACAAGGCCCGGG AACGTATTCACCGCGGCGTGCTGATCCGCGA TTAATAGCTATTCCAGCTTCGTGCAGTCGAG 

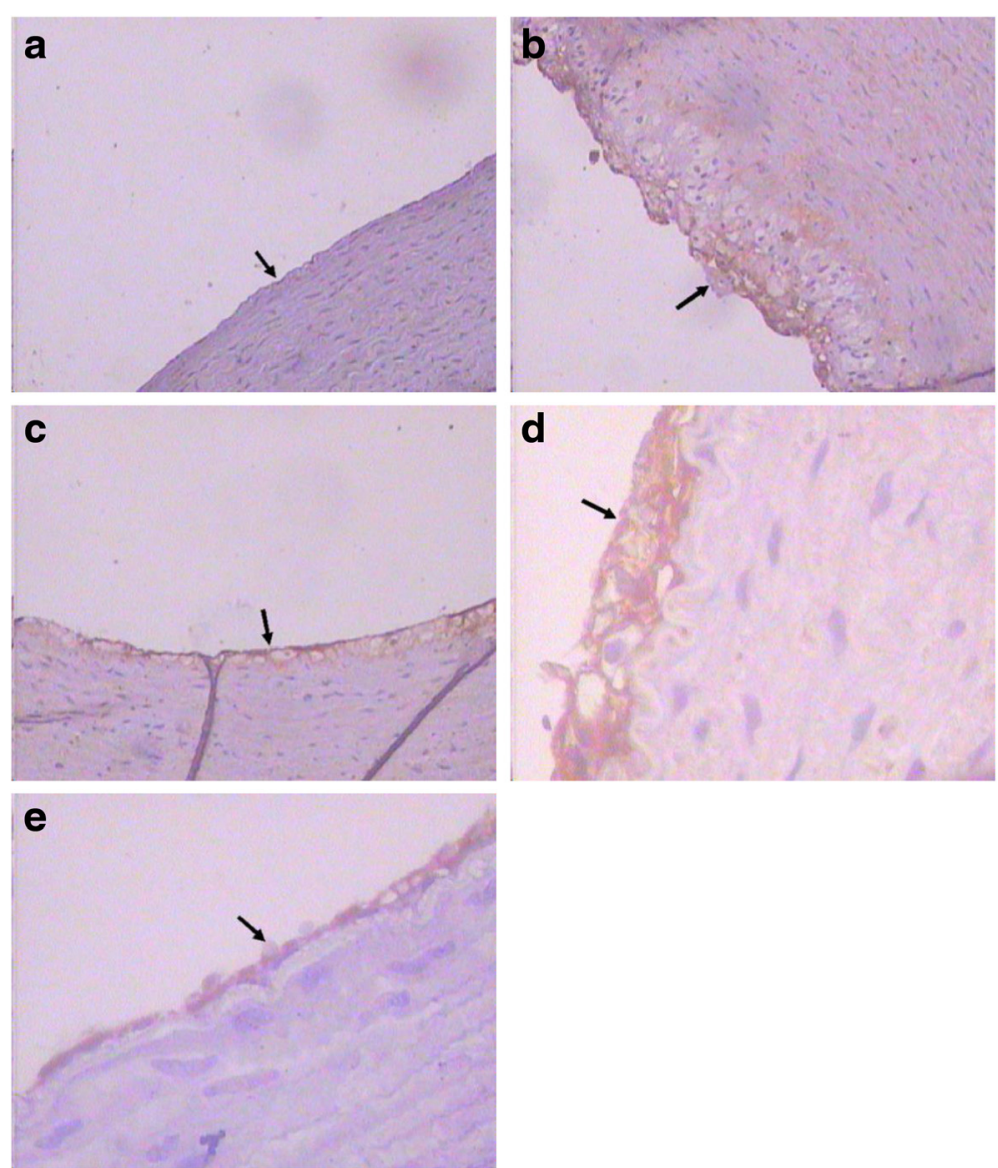

Fig. 2 The effect of TFCW on VCAM-1 levels of aorta in atherosclerotic rabbits. a Normal control; (b) atherosclerotic group; (c) positive control (simvastatin $20 \mathrm{mg} / \mathrm{kg}$ ); (d) low dose TFCW (25 mg/kg); (e) high dose TFCW (50 mg/kg). (Magnification 400x)

TTGCAGACTGCAGTCCGAACTGAGAACAGC TTTCAGAGAATTGCTTGCCTTTGCAGGCTCG CTGCTCGTTGTGCTGCCCATTGTAGCACGTG TGTAGCCCAGGTCATAAGGGGCATGATGACT TGACGTCATCCCCACCTTCCTCCGGTTTGTC ACCGGCAGTCTCATTAGAGTGCCCAACTTAA TGCTGGCAACTAATAACAAGGGTTGCGCTCG TTGCGGGACTTAACCCAACATCTCACGACA CGAGCTGACGACAGCCATGCACCACCTGTCT TAGCGTCCCCGAAGGGAACTTTGTATCTCTA CAAATGGCACTAGATGTCAAGACCTGGTAA GGTTCTTCGCGTTGCTTCGAATTAAACCACA TGCTCCACCGCTTGTGCGGGCCCCCGTCAAT TCCTTTGAGTTTCAACCTTGCGGTCGTACTC CCCAGGCGGAGTGCTTAATGCGTTAGCTGCA GCACTGAGAGGCGGAAGCCTCCCAACACTT AGCACTCATCGTTTACGGCATGGACTACCAG GGTATCTAATCCTGTTCGCTACCCATGCTTTC GAGCCTCAGCGTCAGTTGCAGACCAGAGAG CCGCCTTCGCCACTGGTATTCTTCCATATAT CTACGCATTCCACCGCTACACATGGAGTTCTA
CTCTCCTCTTCTGCACTCAAGAAAAACAGTT TCCGATGCAATTCCTCGGTTAAGCCGAGGGC TTTCACATCAGACTTATTCTTCCGCCTGCGCT CGCTTTACGCCCAATAAATCCGGACAACGCT TGCCACCTACGTATTACCGCGGCTGCTGGCA CGTAGTTAGCCGTGACTTTCTGGTTGATTAC CGTCAAATAAAGGCCAGTTACTACCTCTATCC TTCTTCACCAACAACAGAGCTTTACGGTCCG AAAACCTTCTTCACTCACGCGGCGTTGCTCC ATCAGACTTGCGTCCATTGTGGAAGATTCCC TACTGCTGCCTCCCGTANGAGTTTGGGCCGT GTCTCAGTCCCAATGTGGCCGATCAGTCTCT CAACTCGGCTATGCATCACTGCCTTGGTAGG CCGTTACCTTACCAACTAGCTAATGCACCGC GGGTCCATCCTTTAGCGACAGCTTGCGCCGC CTTTTAAAAGCTGTTCATGCGAACTGCTTTC TTATCCGGTATTAGCACCTGTTTCCAAGTGGT ATCCCAGACTTAAGGGCAGGTTCCCCACGTG TTACTCACCCATCCGCCGCTCGCTTTCCCAG CGTCCTCACCGAAGTGATTCTGCTGGTTCCG CTCGCTCGACTTGCATGTATTAG 
Table 3 Biochemical characteristics of LAB isolated from TFCW

\begin{tabular}{|c|c|c|c|c|c|c|c|}
\hline Tests & 1 & 2 & 3 & 4 & 5 & 6 & 7 \\
\hline Catalase test & - & - & - & - & - & - & - \\
\hline Oxidase test & - & - & - & - & - & - & - \\
\hline Indole test & - & - & - & - & - & - & - \\
\hline Trehalose & - & + & + & + & + & + & + \\
\hline Amygdalin & - & - & - & + & + & + & + \\
\hline Common temperature (in air) & + & + & + & + & + & + & - \\
\hline $45^{\circ} \mathrm{C}$ (in air) & - & - & - & - & + & + & - \\
\hline $15^{\circ} \mathrm{C}$ (in air) & - & - & - & + & + & + & + \\
\hline Growth in $6.5 \% \mathrm{NaCl}$ & - & - & - & - & - & - & - \\
\hline Grouth in pH 9.6 & - & - & - & - & - & - & - \\
\hline Grouth in $\mathrm{pH} 4.5$ & + & + & + & + & + & + & + \\
\hline Aerogenesis by sodium gluconate fermentation & + & $\mathrm{N}$ & - & + & N & - & - \\
\hline Sorbitol & - & - & - & + & + & - & - \\
\hline Glucose & + & + & + & + & + & + & + \\
\hline Mannose & - & - & + & + & + & + & + \\
\hline Arabinose & + & - & - & - & - & - & - \\
\hline Esculine & - & + & - & + & + & + & + \\
\hline 16S rDNA,16S rRNA sequence homology level (\%) & 99 & 99 & 100 & 99 & 99 & 99 & 99 \\
\hline Melezitose & - & - & - & + & + & + & - \\
\hline Fructose & + & - & + & + & + & + & + \\
\hline Salicin & - & - & - & + & + & + & + \\
\hline Sodium gluconate & + & - & - & + & + & - & - \\
\hline Ribose & + & - & + & + & + & + & + \\
\hline GelatinDeliquescence test & - & - & - & - & - & - & - \\
\hline Xylose & + & - & - & - & - & - & + \\
\hline Rhamnose & - & - & - & - & - & - & - \\
\hline Maltose & + & + & - & + & + & + & + \\
\hline Lactose & - & + & + & + & + & + & + \\
\hline Raffinose & - & + & - & - & + & - & - \\
\hline Aerogenesisbyglucose fermentation & + & - & N & N & N & N & N \\
\hline Melibiose & + & - & - & - & + & - & - \\
\hline Galactose & + & + & + & + & + & + & + \\
\hline Mannitol & - & + & - & + & + & - & + \\
\hline Sucrose & - & + & - & + & + & + & + \\
\hline Cellobiose & - & - & - & + & + & + & + \\
\hline
\end{tabular}

Note: + positive;-negative; Not performed

3. L. helveticus:

TTAGACGGCTCCTTCCCGAAGGTTAGGCCA CCGGCTTTGGGCATTGCAGACTTCCATG GTGTGACGGGCGGTGTGTACAAGGCCCGG GAACGTATTCACCGCGGCGTTCTGATCCGCG ATTACTAGCGATTCCAGCTTCGTGCAGTCGA GTTGCAGACTGCAGTCCGAACTGAGAACA GCTTTCAGAGATTCGCTTGCCTTCGCAGGC TCGCTTCTCGTTGTACTGTCCATTGTAGCAC
GTGTGTAGCCCAGGTCATAAGGGGCATGAT GACTTGACGTCATCCCCACCTTCCTCCGGTT TGTCACCGGCAGTCTCATTAGAGTGCCCAAC TTAATGCTGGCAACTAATAACAAGGGTTGCG CTCGTTGCGGGACTTAACCCAACATCTCACG ACACGAGCTGACGACAGCCATGCACCACCTG TCTTAGCGTCCCCGAAGGGAACTCCTAATCT CTTAGGATGGCACTAGATGTCAAGACCTGGT AAGGTTCTTCGCGTTGCTTCGAATTAAACCA 
CATGCTCCACCGCTTGTGCGGGCCCCCGTCA ATTCCTTTGAGTTTCAACCTTGCGGTCGTAC TCCCCAGGCGGAGTGCTTAATGCGTTAGCTG CAGCACTGAGAGGCGGAAACCTCCCAACACT TAGCACTCATCGTTTACGGCATGGACTACCA GGGTATCTAATCCTGTTCGCTACCCATGCTTT CGAGCCTCAGCGTCAGTTGCAGACCAGAGA GTCGCCTTCGCCACTGGTGTTCTTCCATATA TCTACGCATTCCACCGCTACACATGGAGTTCC ACTCTCCTCTTCTGCACTCAAGAAAAACAGT TTCCGATGCAGTTCCTCGGTTAAGCCGAGGG CTTTCACATCAGACTTATTCTTCCGCCTGCGC TCGCTTTACGCCCAATAAATCCGGACAACGC TTGCCACCTACGTATTACCGCGGCTGCTGGC ACGTAGTTAGCCGTGACTTTCTGGTTGATTA CCGTCAAATAAAGGCCAGTTACTACCTCTAT CCTTCTTCACCAACAACAGAGCTTTACGATC CGAAAACCTTCTTCACTCACGCGGCGTTGCT CCATCAGACTTGCGTCCATTGTGGAAGATTC CCTACTGCTGCCTCCCGTAGGAGTTTGGGCC GTGTCTCAGTCCCAATGTGGCCGTTCAGTCT CTCAACTCGGCTATGCATCATTGCCTTGGTA AGCCGTTACCTTACCAACTAGCTAATGCACC GCGGGGCCATCCCATAGCGACAGCTTACGCC GCCTTTTATAAGCTGATCATGCGATCTGCTTT CTTATCCGGTATTAGCACCTGTTTCCAAGTG GTATCCCAGACTATGGGGCAGGTTCCCCACG TGTTACTCACCCATCCGCCGCTCGCGTCCCC AGCATCATTACCGAAGTAAATCTGCTGGTTCT GCTCGCTCGACTGCATGTAT

4. L. casei:

TAGACGGCTCGCTCCCTAAAAGGGTTACGCC ACCGGCTTCGGGTGTTACAAACTCTCATGGT GTGACGGGCGGTGTGTACAAGGCCCGGGAA CGTATTCACCGCGGCGTGCTGATCCGCGATT ACTAGCGATTCCGACTTCGTGTAGGCGAGTT GCAGCCTACAGTCCGAACTGAGAATGGCTTT AAGAGATTAGCTTGACCTCGCGGTCTCGCAA CTCGTTGTACCATCCATTGTAGCACGTGTGTA GCCCAGGTCATAAGGGGCATGATGATTTGAC GTCATCCCCACCTTCCTCCGGTTTGTCACCG GCAGTCTTACTAGAGTGCCCAACTAAATGCT GGCAACTAGTCATAAGGGTTGCGCTCGTTGC GGGACTTAACCCAACATCTCACGACACGAGC TGACGACAACCATGCACCACCTGTCATTTTG CCCCCGAAGGGGAAACCTGATCTCTCAGGTG ATCAAAAGATGTCAAGACCTGGTAAGGTTCT TCGCGTTGCTTCGAATTAAACCACATGCTCC ACCGCTTGTGCGGGCCCCCGTCAATTCCTTT GAGTTTCAACCTTGCGGTCGTACTCCCCAGG CGGAATGCTTAATGCGTTAGCTGCGGCACTG AAGGGCGGAAACCCTCCAACACCTAGCATTC ATCGTTTACGGCATGGACTACCAGGGTATCT AATCCTGTTCGCTACCCATGCTTTCGAGCCT
CAGCGTCAGTTACAGACCAGACAGCCGCCTT CGCCACTGGTGTTCTTCCATATATCTACGCAT TTCACCGCTACACATGGAGTTCCACTGTCCT CTTCTGCACTCAAGTTTCCCAGTTTCCGATG CGCTTCCTCGGTTAAGCCGAGGGCTTTCACA TCAGACTTAAAAAACCGCCTGCGCTCGCTTT ACGCCCAATAAATCCGGATAACGCTTGCCAC CTACGTATTACCGCGGCTGCTGGCACGTAGT TAGCCGTGGCTTTCTGGTTGGATACCGTCAC GCCGACAACAGTTACTCTGCCGACCATTCTT CTCCAACAACAGAGTTTTACGACCCGAAAGC CTTCTTCACTCACGCGGCGTTGCTCCATCAG ACTTGCGTCCATTGTGGAAGATTCCCTACTG CTGCCTCCCGTANNAGTTTGGGCCGTGTCTC AGTCCCAATGTGGCCGATCAACCTCTCAGTT CGGCTACGTATCATCGCCTTGGTGAGCCATT ACCTCACCAACTAGCTAATACGCCGCGGGTC CATCCAAAAGCGATAGCTTACGCCATCTTTC AGCCAAGAACCATGCGGTTCTTGGATCTATG CGGTATTAGCATCTGTTTCCAAATGTTATCCC CCACTTAAGGGCAGGTTACCCACGTGTTACT CACCCGTCCGCCACTCGTTCCATGTTGAATC TCGGTGCAAGCACCGATCATCAACGAGAACT CGTTCGACTGCATGTATAGC

5. L. plantarum:

GGCGTGCCTAATACATGCAAGTCGAACGAAC TCTGGTATTGATTGGTGCTTGCATCATGATT TACATTTGAGTGAGTGGCGAACTGGTGAGT AACACGTGGGAAACCTGCCCAGAAGCGGGG GATAACACCTGGAAACAGATGCTAATACCGC ATAACAACTTGGACCGCATGGTCCGAGTTTG AAAGATGGCTTCGGCTATCACTTTTGGATGG TCCCGCGGCGTATTAGCTAGATGGTGGGGTA ACGGCTCACCATGGCAATGATACGTAGCCGA CCTGAGAGGGTAATCGGCCACATTGGGACTG AGACACGGCCCAAACTCCTACGGGAGGCAGC AGTAGGGAATCTTCCACAATGGACGAAAGTC TGATGGAGCAACGCCGCGTGAGTGAAGAAG GGTTTCGGCTCGTAAAACTCTGTTGTTAAAG AAGAACATATCTGAGAGTAACTGTTCAGGTA TTGACGGTATTTAACCAGAAAGCCACGGCTA ACTACGTGCCAGCAGCCGCGGTAATACGTAG GTGGCAAGCGTTGTCCGGATTTATTGGGCGT AAAGCGAGCGCAGGCGGTTTTTTAAGTCTGA TGTGAAAGCCTTCGGCTCAACCGAAGAAGTG CATCGGAAACTGGGAAACTTGAGTGCAGAAG AGGACAGTGGAACTCCATGTGTAGCGGTGAA ATGCGTAGATATATGGAAGAACACCAGTGGC GAAGGCGGCTGTCTGGTCTGTAACTGACGCT GAGGCTCGAAAGTATGGGTAGCAAACAGGAT TAGATACCCTGGTAGTCCATACCGTAAACGAT GAATGCTAAGTGTTGGAGGGTTTCCGCCCTT CAGTGCTGCAGCTAACGCATTAAGCATTCCG CCTGGGGAGTACGGCCGCAAGGCTGAAACTC 
AAAGGAATTGACGGGGGCCCGCACAAGCGG TGGAGCATGTGGTTTAATTCGAAGCTACGCG AAGAACCTTACCAGGTCTTGACATACTATGC AAATCTAAGAGATTAGACGTTCCCTTCGGGG ACATGGATACAGGTGGTGCATGGTTGTCGTC AGCTGGTGTCGTGAGATGTTGGGTTAAGTCC CGCAACGAGCGCAACCCTTATTATCAGTTGC CACCATTAAGTTGGGCACTCTGGTGAGACTG CCGGTGACAAACCGGAGGAAGGTGGGGATG ACGTCAAATCATCATGCCCCTTATGACCTGGG CTACACACGTGCTACAATGGATGGTACAAGG AGTTGCGAACTCGCGAGAGTAAGCTAATCTC TTAAAGCCATTCTCAGTTCGGATTGTAGGCT GCAACTCGCCTACATGAAGTCGGAATCGCTA GTAATCGCGGATCAGCATGCCGCGGTGAATA CGTTCCCGGGCCTTGTACACACCGCCCGTCA CACCATGAGAGTTTGTAACACCCAAAGTCGG TGGGGTAACCTTTTAGGAACCAGCCGCCTAA GGTGG

6. L. kefiri:

CTTAGACGGCTGGTCCCCGAAGGTTACCTCA CCGGCTTTGGGTGTTACAAACTCTCATGGTG TGACGGGCGGTGTGTACAAGGCCCGGGAAC GTATTCACCGTGGCATGCTGATCCACGATTAC TAGCGATTCCAACTTCATGCAGGCGAGTTGC AGCCTGCAATCCGAACTGAGAACGGCTTTAA GAGATTAGCTTGACCTCGCGGTTTCGCGACT CGTTGTACCGTCCATTGTAGCACGTGTGTAG CCCAGGTCATAAGGGGCATGATGATTTGACG TCATCCCCACCTTCCTCCGGTTTGTCACCGG CAGTCTTGCTAGAGTGCCCAACTGAATGCTG GCAACTAACAATAAGGGTTGCGCTCGTTGCG GGACTTAACCCAACATCTCACGACACGAGCT GACGACAACCATGCACCACCTGTCATTCTGT CCCCGAAGGGAACGCCTAATCTCTTAGGTTG GCAGAAGATGTCAAGACCTGGTAAGGTTCTT CGCGTAGCATCGAATTAAACCACATGCTCCA CCGCTTGTGCGGGCCCCCGTCAATTCCTTTG AGTTTCAACCTTGCGGTCGTACTCCCCAGGC GGAGTGCTTAATGCGTTAGCTGCAGCACTGA AGGGCGGAAACCCTCCAACACTTAGCACTCA TCGTTTACGGCATGGACTACCAGGGTATCTA ATCCTGTTCGCTACCCATGCTTTCGAGCCTC AGCGTCAGTTACAGACCAGACAGCCGCCTTC GCCACTGGTGTTCTTCCATATATCTACGCATT TCACCGCTACACATGGAGTTCCACTGTCCTC TTCTGCACTCAAGTCTCCTGGTTTCCGATGC ACTTCTCCGGTTAAGCCGAAGGCTTTCACAT CAGACCTAAGAAACCGCCTGCGCTCGCTTTA CGCCCAATAAATCCGGACAACGCTTGCCACC TACGTATTACCGCGGCTGCTGGCACGTAGTT AGCCGTGGCTTTCTGGTTGGATACCGTCAAG ATGTCAACAGTTACTCTGACACCTGTTCTTCT CCAACAACAGAGTTTTACGAGCCGAAACCCT
TCATCACTCACGCGGCGTTGCTCCATCAGAC TTTCGTCCATTGTGGAAGATTCCCTACTGCT GCCTCCCGTAGGAGTTTGGGCCGTGTCTCAG TCCCAATGTGGCCGATTACCCTCTCAGGTCG GCTACGTATCATTGCCTTGGTAGGCCATTACC TTACCAACAAGCTAATACGCCGCGGGTCCAT CCTAAAGTGATAGCCGAAGCCATCTTTTAAA CCAAAACCATGTGGTTTTGGTTGTTATACGG TATTAGCACCTGTTTCCAAGTGTTATCCCCT ACTTCAAGGGCAGGTTACCCACGTGTTACTC ACCAGTTCGCCACTCGTTTCGTGTTAAATCAT TTAAATGCAAGCATCTAAAATCAATAACGGAA ACGCGTTCGACTTGCATGTAT

7. Lactococcus lactic: GGTCTTACCTTAGGAAGCGCCCTCCTTGCGG TTAGGCAACCTACTTCGGGTACTCCCAACTC CCGTGGTGTGACGGGCGGTGTGTACAAGGC CCGGGAACGTATTCACCGCGGCGTGCTGATC CGCGATTACTAGCGATTCCGACTTCATGTAG GCGAGTTGCAGCCTACAATCCGAACTGAGAA TGGTTTTAAGAGATTAGCTAAACATCACTGTC TCGCGACTCGTTGTACCATCCATTGTAGCAC GTGTGTAGCCCAGGTCATAAGGGGCATGATG ATTTGACGTCATCCCCACCTTCCTCCGGTTTA TCACCGGCAGTCTCGTTAGAGTGCCCAACTT AATGATGGCAACTAACAATAGGGGTTGCGCT CGTTGCGGGACTTAACCCAACATCTCACGAC ACGAGCTGACGACAACCATGCACCACCTGTA TCCCGTGTCCCGAAGGAACTTCCTATCTCTAG GAATAGCACGAGTATGTCAAGACCTGGTAAG GTTCTTCGCGTTGCTTCGAATTAAACCACAT GCTCCACCGCTTGTGCGGGCCCCCGTCAATT CCTTTGAGTTTCAACCTTGCGGTCGTACTCC CCAGGCGGAGTGCTTATTGCGTTAGCTGCGA TACAGAGAACTTATAGCTCCCTACATCTAGCA CTCATCGTTTACGGCGTGGACTACCAGGGTA TCTAATCCTGTTTGCTCCCCACGCTTTCGAG CCTCAGTGTCAGTTACAGGCCAGAGAGCCGC TTTCGCCACCGGTGTTCCTCCATATATCTACG CATTTCACCGCTACACATGGAATTCCACTCTC CTCTCCTGCACTCAAGTCTACCAGTTTCCAAT GCATACAATGGTTGAGCCACTGCCTTTTACA CCAGACTTAATAAACCACCTGCGCTCGCTTT ACGCCCAATAAATCCGGACAACGCTCGGGAC CTACGTATTACCGCGGCTGCTGGCACGTAGT TAGCCGTCCCTTTCTGGGTAGTTACCGTCAC TTGATGAGCTTTCCACTCTCACCAACGTTCT TCTCTACCAACAGAGTTTTACGATCCGAAAAC CTTCTTCACTCACGCGGCGTTGCTCGGTCAG ACTTTCGTCCATTGCCGAAGATTCCCTACTG CTGCCTCCCGTANGAGTTTGGGCCGTGTCTC AGTCCCAATGTGGCCGATCACCCTCTCAGGT CGGCTATGTATCATCGCCTTGGTGAGCCTTTA CCTCACCAACTAGCTAATACAACGCGGGATC 
ATCTTTGAGTGATGCAATTGCATCTTTCAAAC TTAAAACTTATGTTTAAAGTTGTTATGCGGTA TTAGCATTCGTTTCCAAATGTTGTCCCCCGCT CAAAGGCAGATTCCCCACGCGTTACTCACCC GTTCGCTGCTCTTCAAATTGGTGCAAGCACC AATCTTCATCGCTCAACTTGCATGATTAG

\section{Identification of probiotic yeasts}

We identified two yeast isolates to be S. unisporus and $I$. orientalis based on morphological characteristics, physiological and biochemical tests and 26S rDNAD1/D2 sequence homology with sequences AY 707865 and EU 019220 in Genbank. All the test results and sequence homology were shown in Table 4, while all of the sequences were as follows:

1. S. unisporus: TGCATATTCAATAAGCGGAGGAAAAGAAACC AACCGGGATTGCCTTAGTAACGGCGAGTGAA GCGGCAAAAGCTCAAATTTGAAATCTAGTAC CTTCGGTGCTCGAGTTGTAATTTGTAGAGGG ATACTTTGGGGCCGTTCCTTGTCTATGTTCCT TGGAACAGGACGTCATAGAGGGTGAGAATCC CGTGTGGCGAGGAGTGCGGTTCTATGTAAAG TGCCTTCGAAGAGTCGAGTTGTTTGGGAATG CAGCTCTAAGTGGGTGGTAAATTCCATCTAA AGCTAAATATTGGCGAGAGACCGATAGCGAA CAAGTACAGTGATGGAAAGATGAAAAGAACT TTGAAAAGAGAGTGAAAAAGTACGTGAAATT GTTGAAAGGGAAGGGCATTTGATCAGACATG GTGTTTTGCGCCCTCTGCTCCTTGTGGGTGG GGGAATCTCGCAGCTCACTGGGCCAACATCA GTTTTGGTGGTCGGATAAATCCGTAGGAATG TGGCTTGCCTCGGCAAGTGTTATAGCCTGCG GGAATACGGCCAGCTGGGACTGAGGACTGCC ACTTTTGTCAAGGATGTTGGCATAATGGTTAT ATGCCGCCCGTCTTGAAACACGGACCAA

2. I. orientalis: GCATATCAATAAGCGGAGGAAAAGAAACCAA CAGGGATTGCCTCAGTAGCGGCGAGTGAAGC GGCAAGAGCTCANATTTGAAATCGTGCTTTG CGGCACGAGTTGTAGATTGCAGGTTGGAGTC TGTGTGGAAGGCGGTGTCCAAGTCCCTTGGA ACAGGGCGCCCAGGAGGGTGAGAGCCCCGT GGGATGCCGGCGGAAGCAGTGAGGCCCTTC TGACGAGTCGAGTTGTTTGGGAATGCAGCTC CAAGCGGGTGGTAAATTCCATCTAAGGCTAA ATACTGGCGAGAGACCGATAGCGAACAAGTA CTGTGAAGGAAAGATGAAAAGCACTTTGAAA AGAGAGTGAAACAGCACGTGAAATTGTTGAA AGGGAAGGGTATTGCGCCCGACATGGGGATT GCGCACCGCTGCCTCTCGTGGGCGGCGCTCT GGGCTTTCCCTGGGCCAGCATCGGTTCTTGC TGCAGGAGAAGGGGTTCTGGAACGTGGCTC TTCGGAGTGTTATAGCCAGGGCCAGATGCTG

\section{CGTGCGGGGACCGAGGACTGCGGCCGTGTA GGTCACGGATGCTGGCAGAACGGCGCAACA CCGCCCGTCTTGAAACACGGACCA}

\section{Discussion}

Major finding of the current study is that treatment with TFCW significantly modified lipid profile and reduced CRP, ICAM-1 and VCAM-1 in atherosclerotic rabbit model. Preventive effects of TFCW in atherogenic rabbits were also demonstrated by reduction in VCAM-1 expression and formation of atheromatous plaques on aortic endothelium. In fact, accumulation of cholesterol and lipids leads to foam cell formation, which is regarded as a critical process in development of atherosclerosis [12]. Overwhelmingly strong evidence demonstrated that integrated dysregulation of serum lipidic and inflammatory components in vascular wall contributes to an early and advanced atherosclerotic development [13]. VCAM-1 is a critical mediator of adhesion and uptake of monocytes across the endothelium in the early stages of atherosclerosis development [14], which mediates the assembly of monocytes, macrophages, $\mathrm{T}$ lymphocytes and platelets and their adherence to vascular wall that plays a key role in pathogenesis of atherosclerosis [15]. CRP, a phylogenetically highly conserved plasma protein, is the classical acute phase reactant in humans, and preliminary evidence for interaction of CRP with lipids implicates a possible relationship between CRP and atherosclerosis [16].

In this study, 7 potential probiotic lactobacillus species, including L. casei [17], L. helveticus [18], L. plantarum [19] and L. lactis [20], which are proven probiotics, were identified in the TFCW. These LAB species may be responsible for the protective effect of TFCW against atherosclerosis in atherogenic rabbits. Indeed, $\mathrm{LAB}$ increase immune response [21] and reduce cholesterol [22, 23] both in animal models $[24,25]$ and humans [26]. LAB or LAB with active bile salt hydrolase have been suggested to lower cholesterol through interaction with host bile salt metabolism [27].

In addition, goat milk fermented with Lactocillus fermenterum ME-3 improves antioxidant activities in human blood, thus providing antiatherogenic activity [28]. Consumption of probiotic-containing dairy food reduces cholesterol possibly through degradation of cholesterol, and probiotic lactobacilli and their metabolic by-products lower cholesterol and provide preventive and therapeutic effects against ischemic heart syndromes [6, 29].

We also identified two probiotic yeasts in TFCW. $S$. unisporus is ubiquitously present in fermented milk, cheese and kefir-based milk products and may produce vitamins and interact with $\mathrm{LAB}$, which may enhance LAB growth [30]. S. unisporus contains middle chain fatty acids up to C $14: 0$ to $18: 1$ and produces a high percentage of palmitoleate. Palmitoleic acid, an omega-7 
Table 4 Biochemical characteristics of yeasts isolated from TFCW

\begin{tabular}{|c|c|c|}
\hline Test & $A$ & $B$ \\
\hline Glucose & + & + \\
\hline Galactose & - & - \\
\hline Sucrose & - & - \\
\hline Maltose & - & - \\
\hline Lactose & - & - \\
\hline Raffinose & - & - \\
\hline Arabinose & - & - \\
\hline Cellobiose & - & - \\
\hline Esculin & - & - \\
\hline Fructose & - & - \\
\hline Mannitol & - & - \\
\hline Mannose & - & - \\
\hline Melibiose & - & - \\
\hline Rhamnose & - & - \\
\hline Salicin & - & - \\
\hline Sorbose & - & - \\
\hline Xylose & - & - \\
\hline MushroomCarbohydrates & - & - \\
\hline D-Ribose & - & - \\
\hline Carbon Assimilation tests & - & - \\
\hline Galactose & + & - \\
\hline Sucrose & - & - \\
\hline Maltose & - & - \\
\hline Cellobiose & - & - \\
\hline Trehalose & - & - \\
\hline Lactose & - & - \\
\hline Raffinose & - & - \\
\hline Soluble starch & - & - \\
\hline D-xylose & - & - \\
\hline L-arabinose & - & - \\
\hline L-arabinose & - & - \\
\hline D-ribose & - & - \\
\hline L-Rhamnose & - & - \\
\hline Erythrosealcohol & - & - \\
\hline Adonitol & - & - \\
\hline D-mannitol & - & - \\
\hline Succinate & - & + \\
\hline Citric acid & - & + \\
\hline Inositol & - & - \\
\hline Nitrogen Assimilation test & & - \\
\hline Nitrate & - & - \\
\hline Hydrochloric cadaverine & + & + \\
\hline
\end{tabular}

Table 4 Biochemical characteristics of yeasts isolated from TFCW (Continued)

\begin{tabular}{|c|c|c|}
\hline L-Lysine & + & - \\
\hline $\begin{array}{l}\text { Growth in medium } \\
\text { vitamin free }\end{array}$ & - & + \\
\hline Growth at $37^{\circ} \mathrm{C}$ & + & + \\
\hline Colony size $(\mu \mathrm{m})$ & $(2.5-4.5) \times(3.6-6.0)$ & $(2.4-3.6 \times(3.6-6.0)$ \\
\hline $\begin{array}{l}\text { 26SrDNAD1/ } \\
\text { 2sequencehomology } \\
\text { level (\%) }\end{array}$ & 99 & 99 \\
\hline
\end{tabular}

monounsaturated fatty acid, is a major constituent of human adipose tissues and is considered antioxidant [31]. I.orientalis exhibits a higher tolerance for $\mathrm{pH}$, bile, and heat stress for survival in gastrointestinal environment as a probiotic [32]. I. orientalis commonly exists in cheeses and other fermentation milk products and exhibits ability to scavenge 1,1 diphenyl-2-picrylhydrazyl and to inhibit lipid peroxidation, thus presenting antioxidant activity as a potential probiotic in fermented milk products [33].

Notably, oxidized LDL in vascular wall seems to be a key factor in atherosclerosis, because oxidized LDLs might recruit monocytes and favor their transformation into foam cells through a receptor-mediated intake (scavenger pathway). Moreover, cytotoxic oxidized form of LDLs are likely responsible for endothelial cell damage and macrophage degeneration in atherosclerotic human plaque [34]. Polyunsaturated fat decreases TC and LDL-C by lowering LDL-C production rates and/or increasing LDL clearance rates $[35,36]$. Consequently, omega-3polyunsaturated fatty acid ( $\omega 3$-PUFA) has beneficial effects in preventing atherosclerotic diseases, and a strong positive correlation prevails between intake of saturated fatty acids and an increased incidence of CVD [37]. Therefore, inhibition of oxidation of unsaturated fatty acids is of significance for the prevention of atherosclerosis and/or CVD [38]. Furthermore, hypercholesterolemia is a major risk factor for the development of atherosclerosis [39]. Thus, we speculate that TFCW exerts its anti-atherogenic effect possibly through the identified probiotic $L A B$ and yeasts. However, atherosclerotic effect of each individual LAB was not investigated in this study, and further studies of such effects by each kind of $\mathrm{LAB}$ and the possible underlying mechanisms are necessary.

\section{Conclusions}

In conclusion, current study indicates that $7 \mathrm{LAB}$ and 2 yeasts identified from TFCW and TFCW have significant anti-atherosclerotic potential in atherosclerotic rabbits and may modulate lipid metabolism and protect aorta in the atherosclerotic condition, which might be related to various probiotics acting through reducing the CRP, VCAM-1 and ICAM-1 levels and protecting the aortic endothelium. 


\section{Acknowledgments}

This work was supported by the National Natural Science Foundation of China (No. 30860335).

\section{Author contributions}

XHN designed the experiments, provided oversight at all stages of the project and performed animal model and treatment; CYM prepared TFCW and isolated, purified and identified probiotics; $\mathrm{TM}, \mathrm{MH}, \mathrm{BW}$ and $\mathrm{LA}$ conducted the work, collected and analysed the data; $\mathrm{TM}, \mathrm{MH}$ and BW wrote the manuscript. All authors read and approved the final manuscript.

\section{Competing interests}

The authors declare that they have no competing interests.

\section{Received: 19 December 2015 Accepted: 12 August 2016}

\section{Published online: 24 August 2016}

\section{References}

1. Moran A, Forouzanfar M, Sampson U, Chugh S, Feigin V, Mensah G. The epidemiology of cardiovascular diseases in sub-Saharan Africa: the global burden of diseases, injuries and risk factors 2010 study. Prog Cardiovasc Dis. 2013;56(3):234-9.

2. Kones R. Molecular sources of residual cardiovascular risk, clinical signals, and innovative solutions: relationship with subclinical disease, undertreatment, and poor adherence: implications of new evidence upon optimizing cardiovascular patient outcomes. Vasc Health Risk Manag. 2013;9:617-70.

3. Wang XJ, Nabi XH, Sun Y. Effects of active peptide extracted from cheese whey on the expression of PPAR $\gamma$, mRNA and NF-kB activity in 3 T3-L1 adipocytes. J China Pharm Univ. 2012:43:449-52.

4. Khateeb J, Gantman A, Kreitenberg AJ, Aviram M, Fuhrman B. Paraoxonase 1 (PON1) expression in hepatocytes is upregulated by pomegranate polyphenols: a role for PPAR-gamma pathway. Atherosclerosis. 2010;1:119-25.

5. Kevin DB, Richard SB, Richard LS, Erin EQ, Brittanie MV, Daniel JF, Diana MK, Brian RK, Min-Yu C, William JK, Jeff SV. Acute ingestion of a novel whey-derived peptide improves vascular endothelial responses in healthy individuals: a randomized, placebo controlled trial. Nutr J. 2009:8:34

6. Fijan S. Microorganisms with claimed probiotic properties: an overview of recent literature. Int J Environ Res Public Health. 2014; 11(5):4745-67.

7. Banjoko IO, Adeyanju MM, Ademuyiwa O, Adebawo OO, Olalere RA Kolawole MO, Adegbola IA, Adesanmi TA, Oladunjoye TO, Ogunnowo AA, Shorinola AA, Daropale O, Babatope EB, Osibogun AO, Ogunfowokan DT, Jentegbe TA, Apelehin TG, Ogunnowo O, Olokodana O, Fetuga FY, Omitola M, Okafor LA, Ebohon CL, Ita JO, Disu KA, Ogherebe O, Eriobu SU, Bakare AA. Hypolipidemic effects of lactic acid bacteria fermented cereal in rats. Lipids Health Dis. 2012;11:170.

8. do Lee K, Jang S, Baek EH, Kim MJ, Lee KS, Shin HS, Chung MJ, Kim JE, Lee KO, Ha NJ. Lactic acid bacteria affect serum cholesterol levels, harmful fecal enzyme activity, and fecal water content. Lipids Health Dis. 2009;8:21

9. Prakash S, Urbanska AM. Colon-targeted delivery of live bacterial cell biotherapeutics including microencapsulated live bacterial cells. Biologics. 2008:2(3):355-78.

10. Vijayaraghavan K. Treatment of dyslipidemia in patients with type 2 diabetes. Lipids Health Dis. 2010;9:144.

11. Kandler O, Weiss N. Genus Lactobacillus. In: Sneath PHA, Mair NS, Sharpe ME, Holt JG, editors. Bergey's Manual of Systematic Bacteriology. 2nd ed. Baltimore: Williams and Wilkins; 1986. p. 1209-34.

12. Sengupta B, Narasimhulu CA, Parthasarathy S. Novel technique for generating macrophage foam cells for in vitro reverse cholesterol transport studies. J Lipid Res. 2013;54(12):3358-72.

13. Wei H, Zhang WJ, McMillen TS, Leboeuf RC, Frei B. Copper chelation by tetrathiomolybdate inhibits vascular inflammation and atherosclerotic lesion development in apolipoprotein E-deficient mice. Atherosclerosis. 2012;223(2):306-13.

14. liyama K, Hajra L, liyama M, Li H, DiChiara M, Medoff BD, Cybulsky MI. Patterns of vascular cell adhesion molecule-1 and intercellular adhesion molecule-1 expression in rabbit and mouse atherosclerotic lesions and at sites predisposed to lesion formation. Circ Res. 1999;85(2):199-207.
15. Ley K, Miller Yl, Hedrick CC. Monocyte and macrophage dynamics during atherogenesis. Arterioscler Thromb Vasc Biol. 2011;31(7):1506-16.

16. Aho K. Studies of syphilitic antibodies.IV. Evidence of reactant partner common for C-reactive protein and certain anti-lipoidalantibodies. J Vener Dis. 1969;45(1):13-8.

17. Chen S, Lee Y, Crother TR, Fishbein M, Zhang W, Yilmaz A, Shimada K, Schulte DJ, Lehman TJ, Shah PK, Arditi M. Marked acceleration of atherosclerosis after Lactobacillus casei-induced coronary arteritis in a mouse model of Kawasaki disease. Arterioscler Thromb Vasc Biol. 2012; 32(8):e60-71.

18. Taverniti $\vee$, Guglielmetti S. Health-promoting properties of lactobacillus helveticus. Front Microbiol. 2012;3:392.

19. Kim JY, Kim H, Jung BJ, Kim NR, Park JE, Chung DK. Lipoteichoic acid isolated from Lactobacillus plantarum suppresses LPS-mediated atherosclerotic plaque inflammation. Mol Cells. 2013;35(2):115-24.

20. Rezende RM, Oliveira RP, Medeiros SR, Gomes-Santos AC, Alves AC, Loli FG, Guimarães MA, Amaral SS, da Cunha AP, Weiner HL, Azevedo V, Miyoshi A, Faria AM. Hsp65-producing Lactococcus lactis prevents experimental autoimmune encephalomyelitis in mice by inducing CD4 + LAP+ regulatory T cells. J Autoimmun. 2013;40:45-57.

21. Gill HS, Rutherfurd KJ, Cross ML, Gopal PK. Enhancement of immunity in the elderly by dietary supplementation with the probiotic Bifidobacterium lactis HN019. Am J Clin Nutr. 2001;74(6):833-9.

22. Gilliland SE, Nelson CR, Maxwell C. Assimilation of cholesterol by Lactobacillus acidophilus. Appl Environ Microbiol. 1985:49(2):377-81.

23. Park YH, Kim JG, Shin YW, Kim HS, Kim YJ, Chun T, Kim SH, Whang KY. Effects of Lactobacillus acidophilus 43121 and a mixture of Lactobacillus casei and Bifidobacterium longum on the serum cholesterol level and fecal sterol excretion in hypercholesterolemia-induced pigs. Biosci Biotechnol Biochem. 2008;72(2):595-600.

24. Agerbaek M, Gerdes LU, Richelsen B. Hypocholesterolaemic effect of a new fermented milk product in healthy middle-aged men. Eur J Clin Nutr. 1995; 49(5):346-52.

25. Paik HD, Park JS, Park E. Effects of Bacillus polyfermenticus SCD on lipid and antioxidant metabolisms in rats fed a high-fat and high-cholesterol diet. Biol Pharm Bull. 2005:28(7):1270-4.

26. Simons LA, Amansec SG, Conway P. Effect of Lactobacillus fermentum on serum lipids in subjects with elevated serum cholesterol. Nutr Metab Cardiovasc Dis. 2006;16(8):531-5.

27. De Smet I, De Boever P, Verstraete W. Cholesterol lowering in pigs through enhanced bacterial bile salt hydrolase activity. Br J Nutr. 1998; 79(2):185-94.

28. Mikelsaar M, Zilmer M. Lactobacillus fermentum ME-3- an antimicrobial and antioxidative probiotic. Microb Ecol Health Dis. 2009;21(1):1-27.

29. Xiao JZ, Kondo S, Takahashi N, Miyaji K, Oshida K, Hiramatsu A, Iwatsuki K, Kokubo S, Hosono A. Effects of milk products fermented by Bifidobacterium longum on blood lipids in rats and healthy adult male volunteers. J Dairy Sci. 2003;86(7):2452-61.

30. Marsh AJ, O'Sullivan O, Hill C, Ross RP, Cotter PD. Sequencing-based analysis of the bacterial and fungal composition of kefir grains and milks from multiple sources. PLoS One. 2013;8(7):e69371.

31. Palomäki A, Pohjantähti-Maaroos $H$, Wallenius $M$, Kankkunen $P$, Aro $H$, Husgafvel S, Pihlava JM, Oksanen K. Effects of dietary cold-pressed turnip rapeseed oil and butter on serum lipids, oxidized LDL and arterial elasticity in men with metabolic syndrome. Lipids Health Dis. 2010;9:137.

32. Xiao H, Shao Z, Jiang Y, Dole S, Zhao H. Exploiting Issatchenkia orientalis SD108 for succinic acid production. Microb Cell Fact. 2014;13:121.

33. Moslehi-Jenabian S, Pedersen $L L$, Jespersen $L$. Beneficial effects of probiotic and food borne yeasts on human health. Nutrients. 2010;2(4): 449-73.

34. Ahmed KA, Muniandy S, Ismail IS. N (epsilon)-(carboxymethyl) lysine and coronary atherosclerosis-associated Low density lipoprotein abnormalities in type 2 diabetes: current status. J Clin Biochem Nutr. 2009;44(1):14-27.

35. Micha R, Mozaffarian D. Saturated fat and cardiometabolic risk factors, coronary heart disease, stroke, and diabetes: a fresh look at the evidence. Lipids. 2010;45(10):893-905.

36. Kamaly N, Xiao Z, Valencia PM, Radovic-Moreno AF, Farokhzad OC. Targeted polymeric therapeutic nanoparticles: design, development and clinical translation. Chem Soc Rev. 2012;41(7):2971-3010. 
37. Sabzghabaee AM, Kelishadi R, Jelokhanian H, Asgary S, Ghannadi A, Badri S. Clinical effects of portulaca oleracea seeds on dyslipidemia in obese adolescents: a triple-blinded randomized controlled trial. Med Arch. 2014; 68(3):195-9.

38. Véronique L, Roger AS, Go DM, et al. Heart disease and stroke statistics-2012 update: a report from the American Heart Association. Circulation. 2012; 125(1):e2-e220.

39. Mannu GS, Zaman MJ, Gupta A, Rehman HU, Myint PK. Evidence of lifestyle modification in the management of hypercholesterolemia. Curr Cardiol Rev. 2013:9(1):2-14

Submit your next manuscript to BioMed Central and we will help you at every step:

- We accept pre-submission inquiries

- Our selector tool helps you to find the most relevant journal

- We provide round the clock customer support

- Convenient online submission

- Thorough peer review

- Inclusion in PubMed and all major indexing services

- Maximum visibility for your research

Submit your manuscript at www.biomedcentral.com/submit
C) Biomed Central 